\title{
Evolution of electron spectrum in symmetric two-barrier open nanostructure under the influence of strong electromagnetic field
}

\author{
Tkach M. V., Seti Ju. O. and Voitsekhivska O. M. \\ Chernivtsi National University, 2 Kotsiybynsky St., 58012 Chernivtsi, Ukraine \\ e-mail: ktf@chnu.edu.ua
}

Received: 14.12 .2011

\begin{abstract}
A theory of quasi-stationary spectrum of electrons interacting with electromagnetic field of arbitrary intensity in an open two-barrier resonance tunnel structure is developed using exact solution of the complete Schrödinger equation. It is shown that, besides the quasi-stationary electronic states having usual resonance energies and widths, additional satellite quasi-stationary states appear in the spectrum, with their resonance energies located near the resonance energies of every of the quasi-stationary states at the distances being multiples of an energy fixed by the electromagnetic field frequency.
\end{abstract}

Keywords: quasi-stationary state, resonance tunnel structure, high-frequency electromagnetic field, permeability coefficient

PACS: 73.21.Fg, 73.63.Hs, 73.50.Fq, 78.67.De

UDC: $538.915,544.174 .2$

\section{Introduction}

There has been a surge in investigations of spectra of electrons interacting with electromagnetic fields in resonance tunnel structures (RTSs) with different geometrical designs after creation of first quantum cascade lasers by Faist and Capasso with co-authors [1,2] and, further, quantum cascade detectors $[3,4]$. The RTSs are basic elements of these nanodevices, having unique physical characteristics and operating in the actual terahertz frequency range.

A theory of electronic currents interacting with electromagnetic field in the RTSs regarded as open nanosystems appears to be rather complicated, even for relatively simple models with $\delta$-like potential barriers. In the majority of the relevant theoretical papers [5-9], a so-called small signal approximation has been used. In its frames, only terms linear in the electric intensity of electromagnetic field are maintained in the Hamiltonian and the wave functions. In essence, the permeability coefficient of an RTS and, since, the spectral parameters of quasi-stationary states (QSSs) have been studied in the abovementioned papers within the first approximation of the perturbation theory dependent on time.

In order to take into account the influence of the intensity of high-frequency fields on the spectrum of electrons and their tunnelling through the RTSs, the frames of the perturbation theory have been abandoned for the model of two-level systems, using the approximation of iteration method (see the earlier papers [10, 11] and those published recently [12]). In these papers, one can see no terms quadratic in the field intensity term in the Hamiltonian, though it is essential for strong enough fields and, therefore, so must be taken into account in the wave function, together with the other terms of higher orders in the field, which are produced by linear interaction.

Within the two-level approximation and under the condition of equal resonance widths of the two QSSs between which the quantum transition happens, the author of Ref. [12] has established 
that, under the influence of strong electromagnetic field when the electronic current flows through the two-barrier RTS, the two transparent canals are observed at the energies essentially different from the resonance ones. Nonetheless, the nature of these QSSs has not been explained. Moreover, the condition of strict equality of the resonance widths cannot be satisfied, even for the neighbouring QSSs in the two-barrier RTS.

In the present study we develop a consistent quantum-mechanical theory of electronic transport through the two-barrier RTSs under the influence of high-frequency electromagnetic field with an arbitrary magnitude of electric intensity, which is exactly taken into consideration both in the Hamiltonian of the system and in the wave functions. This has allowed studying the evolution of quasi-stationary spectra of electrons interacting with the high-frequency electromagnetic field and the permeability of the two-barrier RTSs that depends on the frequency and intensity of this field. We have shown that the interaction between the electrons and the electromagnetic field causes arising of satellite QSSs, with all the field harmonics appearing near all of the electron QSSs observed without the field.

\section{Permeability coefficient for the two-barrier RTS in high-frequency electromagnetic field}

A plane, open and symmetric two-barrier RTS (see Fig. 1) is studied here which is subjected to homogeneous high-frequency electromagnetic field with the frequency $\omega$ and the electric intensity $\mathcal{E}$.

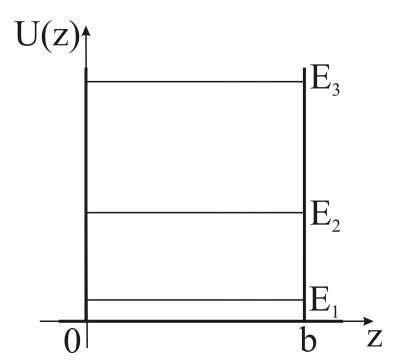

Fig. 1. Energetic scheme for electrons put into a two-barrier RTS.

Let a mono-energetic current of uncoupling electrons (we will only deal with the one-electron problem further on), with the energy $E$, fall on the RTS. It will move along a $z$ axis perpendicular to the RTS planes from the left side. The complete Schrödinger equation for this one-dimensional motion in a quasi-static electromagnetic field is written as

$$
i \hbar \frac{\partial \Psi(E, \omega, z, t)}{\partial t}=\left[-\frac{\hbar^{2}}{2 m} \frac{\partial^{2}}{\partial z^{2}}+U(z)+H(z, t)+\frac{2(e \mathcal{E})^{2}}{m \omega^{2}} \sin (\omega t)^{2}\right] \Psi(E, \omega, z, t) .
$$

Here, the Hamiltonian of the system contains the electron kinetic energy (the first term), the electron potential energy written in a typical $\delta$-barrier approximation (see [5-12]),

$$
U(z)=U \Delta(\delta(z)+\delta(z-b)),
$$

the potential energy of the electron interacting with the electromagnetic field,

$$
H(z, t)=-2 e \mathcal{E} \cos (\omega \mathrm{t})\{z[\theta(\mathrm{z})-\theta(z-b)]+b \theta(z-b)\},
$$

and the potential energy of the field (the last term). Concerning the notation, e and $\mathrm{m}$ are respectively the mass and charge of electron, $U$ and $\Delta$ respectively the height and the width of potential barriers of the two-barrier RTS, and $\theta(z)$ the Heaviside step function.

Ukr. J. Phys. Opt. 2012, V13, №1 
Eq. (1) has the exact solution given by different analytical expressions in different intervals of the $z$ variable:

$$
\Psi(E, \omega, z, t)=e^{-i\left[\omega_{0}+\tilde{\omega}(t)\right] t} \begin{cases}A_{0} e^{i k z}+B_{0} e^{-i k z}, & z<0 \\ \left(A_{1} e^{i k(z+\tilde{z}(t))}+B_{1} e^{-i k(z+\tilde{z}(t))}\right) e^{-i\left[\tilde{\omega}(t) t-\beta \frac{z}{b} \sin (\omega t)\right]}, & 0<z<b \\ A_{2} e^{i[k z+\beta \sin (\omega t)]} . & z>b\end{cases}
$$

Here we have introduced the convenient denotations for the constant parameters and those dependent on time:

$$
\begin{gathered}
\tilde{\omega}(t)=\tilde{\omega}\left[1-\frac{\sin (2 \omega t)}{2 \omega t}\right], \quad \tilde{\omega}=\frac{(e \mathcal{E})^{2}}{\mathrm{~m} \hbar \omega^{2}}, \quad \tilde{z}(t)=\tilde{z} \cos (\omega t), \quad \tilde{z}=\frac{2 e \mathcal{E}}{m \omega^{2}}, \\
\beta=\frac{2 e \mathcal{E} b}{\hbar \omega}, \quad k=\frac{\sqrt{2 m E}}{\hbar}, \quad \omega_{0}=E / \hbar .
\end{gathered}
$$

The wave function $\Psi(E, \omega, z, t)$ may be expressed as a Fourier series over all the harmonics of the main frequency $\omega$ of the field:

$$
\Psi(E, \omega, z, t)=\sum_{n=-\infty}^{+\infty} e^{-i\left[\omega_{0}+n \omega\right] t} \begin{cases}a_{n}\left(A_{0} e^{i k z}+B_{0} e^{-i k z}\right), & z<0 \\ \left(A_{1} b_{k, n} e^{i k z}+B_{1} b_{-k, n} e^{-i k z}\right), & 0<z<b \\ A_{2} c_{n} e^{i k z}, & z>b\end{cases}
$$

with the known coefficients

$$
\begin{gathered}
a_{n}=\frac{1}{2 \pi} \int_{-\pi}^{\pi} \exp \left\{i n x-i \frac{\tilde{\omega}}{\omega}\left[x-\frac{\sin (2 x)}{2}\right]\right\} d x, \\
b_{ \pm k, n}=\frac{1}{2 \pi} \int_{-\pi}^{\pi} \exp \left\{i n x \pm i \tilde{z} k \cos x+i \beta \frac{z}{b} \sin x-i \frac{\tilde{\omega}}{\omega}[2 x-\sin (2 x)]\right\} d x, \\
c_{n}=\frac{1}{2 \pi} \int_{-\pi}^{\pi} \exp \left\{i n x+i \beta \sin x-i \frac{\tilde{\omega}}{\omega}\left[x-\frac{\sin (2 x)}{2}\right]\right\} d x .
\end{gathered}
$$

Now, using the wave function $\Psi(E, \omega, z, t)$ given by Eq. (6) and making the replacement $E \rightarrow E+p \hbar \omega$, we obtain the wave functions $\Psi(E+p \hbar \omega, \omega, z, t)$ that describe the electron states with the energies $E+p \hbar \omega(p= \pm 1, \pm 2 \ldots)$ :

$$
\Psi(E+p \hbar \omega, \omega, z, t)=\sum_{n=-\infty}^{+\infty} e^{-i\left[\omega_{0}+(n+p) \omega\right] t}\left\{\begin{array}{lc}
B_{0}^{p} a_{n} e^{-i k_{p} z}, & z<0 \\
\left(A_{1}^{p} b_{k_{p}, n} e^{i k_{p} z}+B_{1}^{p} b_{-k_{p}, n} e^{-i k_{p} z}\right), & 0<z<b(10) \\
A_{2}^{p} c_{n} e^{i k_{p} z}, & z>b
\end{array}\right.
$$

where

$$
\begin{gathered}
b_{ \pm k_{p}, n}=\frac{1}{2 \pi} \int_{-\pi}^{\pi} \exp \left\{i n x \pm i \tilde{z} k_{p} \cos x+i \beta \frac{z}{b} \sin x-i \frac{\tilde{\omega}}{\omega}[2 x-\sin (2 x)]\right\} d x, \\
k_{p}=\hbar^{-1} \sqrt{2 m(E+p \hbar \omega)} .
\end{gathered}
$$

Here we have accounted for that the electrons got inside the two-barrier RTS into the state with the 
energy $\mathrm{E}$ transit to all the states with the energies $E+p \hbar \omega$, due to interaction with the electromagnetic field. The electrons can leave these states while only leaving the nanostructure.

Now, the complete wave function may be written as a linear superposition of the electron wave functions in the states with all of the possible energies $E+p \hbar \omega(p=0, \pm 1, \ldots)$ :

$$
\Phi(E, \omega, z, t)=\sum_{p=-\infty}^{+\infty} C_{p} \Psi(E+p \hbar \omega, \omega, z, t) \quad\left(C_{0}=1\right)
$$

where the $\Psi(E+p \hbar \omega, \omega, z, t)$ functions are specified by Eqs. (6) and (10) and the unknown coefficients $C_{p= \pm 1, \ldots}$, along with $A_{0,1,2}^{p}$ and $B_{0,1}^{p}$ ones, are determined by the continuity conditions for the wave functions and their current densities at the interfaces between the media written for every harmonics and for any moment of time $t$ :

$$
\left\{\begin{array}{l}
\Phi(E, \omega, 0-\eta, t)=\Phi(E, \omega, 0+\eta, t) \\
\left.\frac{\partial \Phi(E, \omega, z, t)}{\partial z}\right|_{z=0+\eta}-\left.\frac{\partial \Phi(E, \omega, z, t)}{\partial z}\right|_{z=0-\eta}=\left(\frac{2 m U \Delta}{\hbar^{2}}+\frac{2 i \beta}{b} \sin (\omega t)\right) \Phi(E, \omega, 0, t), \\
\Phi(E, \omega, b-\eta, t)=\Phi(E, \omega, b+\eta, t) \\
\left.\frac{\partial \Phi(E, \omega, z, t)}{\partial z}\right|_{z=b+\eta}-\left.\frac{\partial \Phi(E, \omega, z, t)}{\partial z}\right|_{z=b-\eta}=\frac{2 m U \Delta}{\hbar^{2}} \Phi(E, \omega, b, t) .
\end{array}\right.
$$

Due to orthonormality of the harmonics, from the system of Eqs. (14) we obtain the system of infinite number of equations for the unknown coefficients. Restricting the system by rather arbitrarily large, though finite, number of positive $\left(N^{+}\right)$and negative $\left(N^{-}\right)$harmonics and considering that the number of the negative harmonics is confined only by the number of so-called open canals, which satisfy the condition $N^{-}<\left[\omega_{0} / \omega\right]$, we get the finite system of $4\left(N^{-}+N^{+}+1\right)$ equations concerned with the same number of coefficients:

$$
\begin{gathered}
f_{0}^{0}=A_{0} ; \quad f_{1}^{0}=A_{1} ; \quad f_{2}^{0}=A_{2} ; \quad f_{0}^{p \neq 0}=0 ; \quad f_{1}^{p \neq 0}=C_{p} A_{1}^{p} ; \quad f_{2}^{p \neq 0}=C_{p} A_{2}^{p} ; \\
g_{0}^{0}=B_{0} ; \quad g_{1}^{0}=B_{1} ; \quad g_{0}^{p \neq 0}=C_{p} B_{0}^{p} ; \quad g_{1}^{p \neq 0}=C_{p} B_{1}^{p} .
\end{gathered}
$$

They determine the complete wave function in terms of the functions corresponding to the waves that move to the right $(\rightarrow)$ or left $(\leftarrow)$, with respect to the primary direction. Finally, we obtain

$$
\begin{gathered}
\Phi(E, \omega, z, t)=\sum_{p=-N^{-}}^{N^{+}}\left[\Psi^{\rightarrow}(E+p \hbar \omega, \omega, z, t)+\Psi^{\leftarrow}(E+p \hbar \omega, \omega, z, t)\right]= \\
=\sum_{p=-N^{-}}^{N^{+}} \sum_{n=-\infty}^{+\infty} e^{-i\left[\omega_{0}+(n+p) \omega\right] t}\left\{\begin{array}{lc}
a_{n}\left(f_{0}^{p} e^{i k_{p} z}+g_{0}^{p} e^{-i k_{p} z}\right), & z<0 \\
\left(f_{1}^{p} b_{k_{p}, n} e^{i k_{p} z}+g_{1}^{p} b_{-k_{p}, n} e^{-i k_{p} z}\right), & 0<z<b \\
c_{n} f_{2}^{p} e^{i k_{p} z} . & z>b
\end{array}\right.
\end{gathered}
$$

It is clear that the law of preservation for the complete current density through the all open canals must be fulfilled inside the system:

$$
J^{\rightarrow}(E, \omega, z=0)=\sum_{p=-N^{-}}^{N^{+}} J^{\leftarrow}(E+p \hbar \omega, \omega, z=0)+\sum_{p=-N^{-}}^{N^{+}} J^{\rightarrow}(E+p \hbar \omega, \omega, z=b),
$$

where $J^{\rightarrow}(E, \omega, 0), \quad J^{\leftarrow}(E+n \hbar \omega, \omega, 0)$ and $J^{\rightarrow}(E+n \hbar \omega, \omega, b)$ are respectively the densities of the falling current, the reflected one and that which has penetrated through the open canals of the 
two-barrier RTS. They are given by the expression

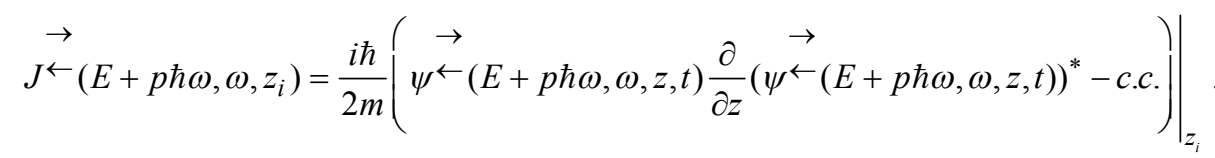

Now, the complete permeability coefficient for the two-barrier RTS can be expressed as a sum

$$
D(E, \omega)=\sum_{p=-N^{-}}^{N^{+}} D_{p}(E+p \hbar \omega, \omega),
$$

with the partial term

$$
D_{p}(E+p \hbar \omega, \omega)=\frac{J^{\rightarrow}(E+p \hbar \omega, \omega, b)}{J^{\rightarrow}(E, \omega, 0)}=\frac{k_{p}}{k}\left|\frac{f_{2}^{p}}{A_{0}}\right|^{2}
$$

describing the probability of the event that the current with the energy $E$ falling at the RTS would exit the nanostructure through the pth open canal with the energy $E+p \hbar \omega$.

The permeability coefficient for the two-barrier RTS obtained by us and given by Eq. (19) enables specify the main QSS and an arbitrary number of the satellite QSSs of electrons interacting with the high-frequency electromagnetic field.

\section{Properties of quasi-stationary electron spectrum of the two-barrier RTS under the influence of electromagnetic field}

Using the theory developed above, we have calculated the permeability coefficient for $\mathrm{In}_{0.52} \mathrm{Al}_{0.48} \mathrm{As} / \mathrm{In}_{0.53} \mathrm{Ga}_{0.47} \mathrm{As}$ a two-barrier RTS, which is often investigated experimentally. Its physical and geometrical parameters are as follows: $m=0.043 m_{e}, U=516 \mathrm{meV}, b=18 \mathrm{~nm}$, and $\Delta=9 \mathrm{~nm}$.

The results of calculations for the permeability coefficient $D$ as a function of the electron energy $\mathrm{E}$ at different magnitudes of non-resonant electromagnetic field energies $\Omega=\hbar \omega$ are shown in Fig. 2 for different magnitudes of shift energies $U_{a c}=e \boldsymbol{E} b$ caused by the electric intensity $\boldsymbol{E}$ of this high-frequency field. It is clear from the inset of Fig. 2a that, if the interaction with the electromagnetic field is absent, the main electron states are quasi-stationary. The spectrum is characterised by the resonance energies $E_{n}$ and the widths $\Gamma_{n}$ determined, as it is known, by the position of maxima and the widths of nth Lorentzian curves, respectively.

The interaction between the electron and the electromagnetic field of an arbitrary (even small) intensity $\mathcal{E}$ and energy $\Omega$ causes the appearance of satellite QSSs, with the resonance energies $E_{n^{(p)}}=E_{n}+p \Omega\left(-N^{-} \leq p \leq N^{+}\right)$located near every of the main states with the resonance energies $E_{n}$.

From Fig. 2 one can see that the maxima of the permeability coefficient for the high$(n, p=1,2, \ldots)$ and low-frequency $(n, p=-1,-2, \ldots)$ non-resonance satellite QSSs of any of the $n$th main QSSs decrease when the order number $p$ increases, irrespective of the intensity $\varepsilon$ or the equivalent energy shift $U_{a c}$ and the field energy $\Omega$. When the resonance energy (n, p) of the satellite QSS gets into resonance with the resonance energy of the $n^{\prime}$ th QSS (for example, $E_{1}+2 \Omega \cong E_{3}$ - see Fig. 2b), the permeability coefficient of this satellite QSS sharply increases and can become larger than that for the satellite states with smaller frequencies $\left(D\left(E_{1}+2 \Omega\right)>D\left(E_{1}+\Omega\right)\right)$. 

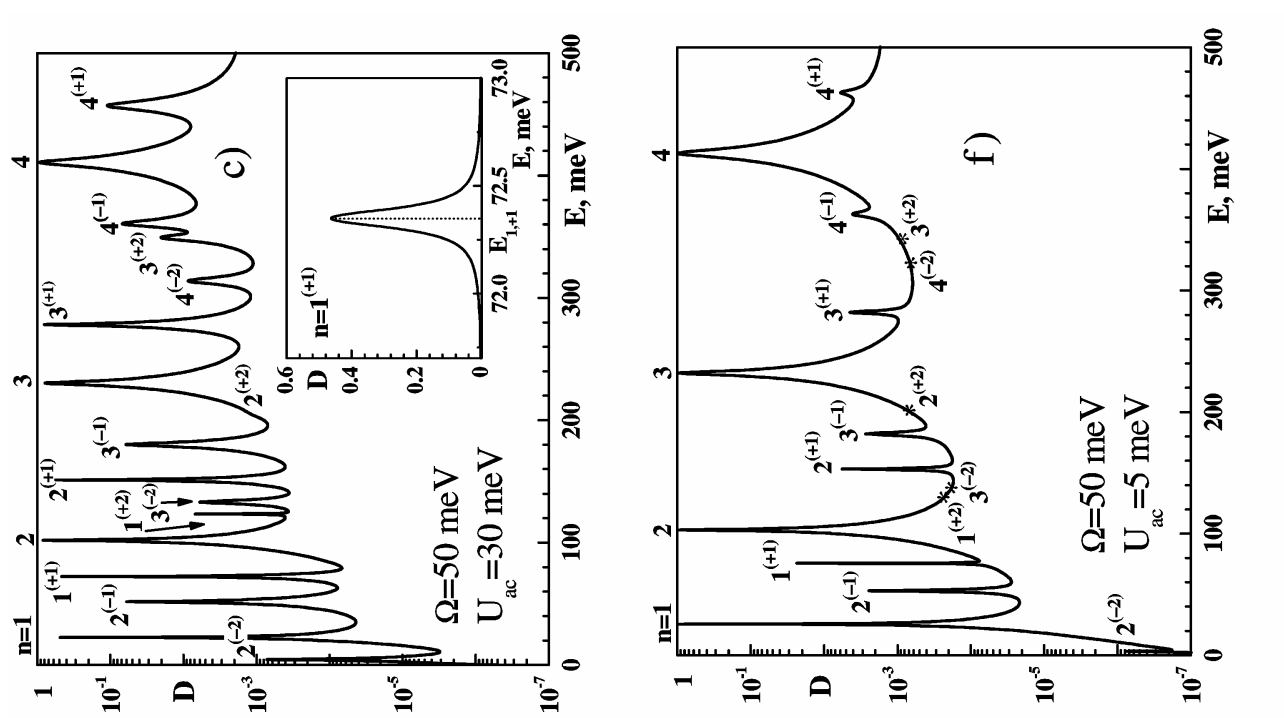

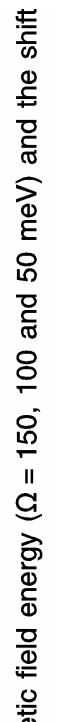
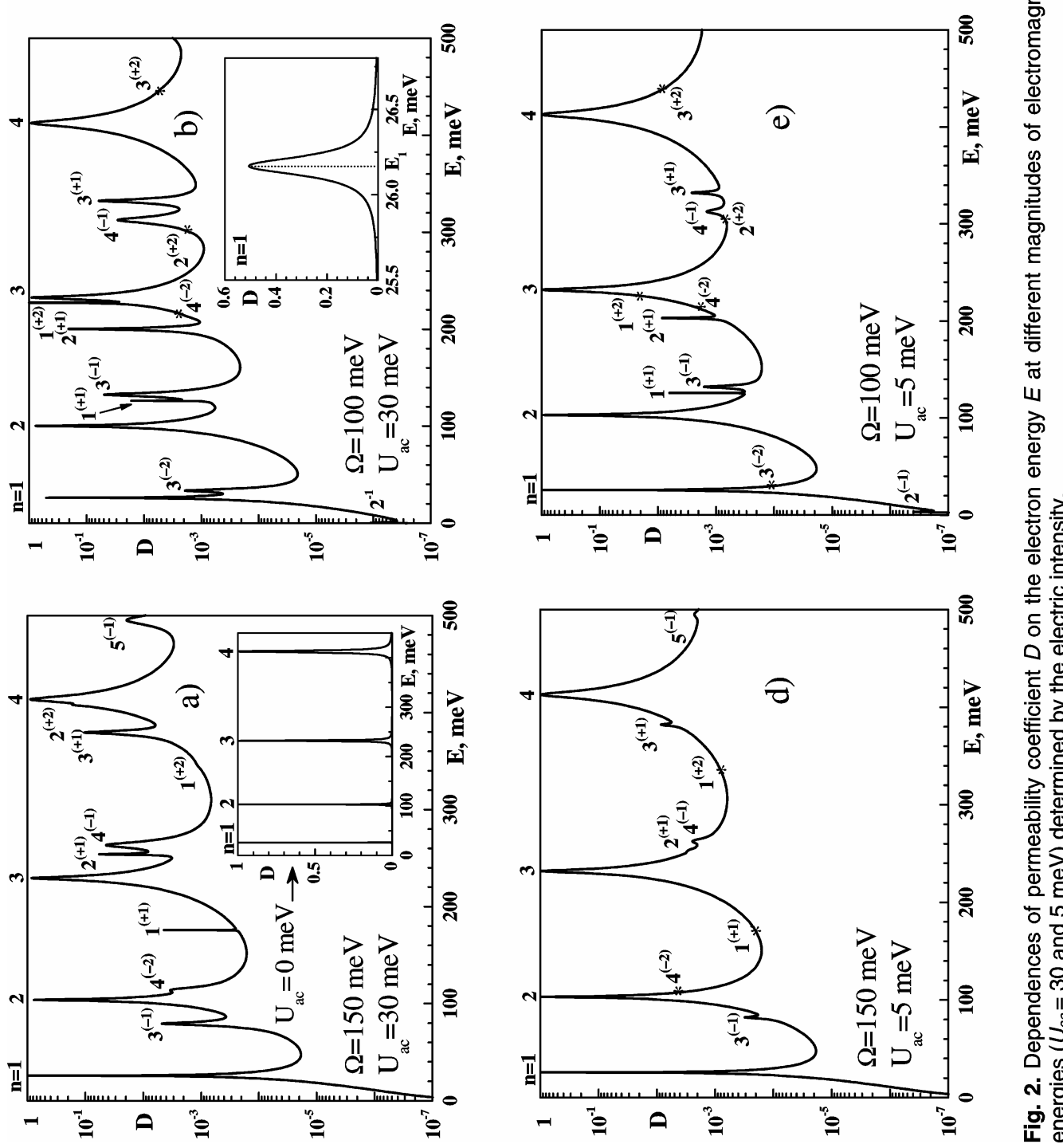

Ukr. J. Phys. Opt. 2012, V13, №1 
The shape of dependence of the permeability coefficient of the non-resonance satellite QSSs (extracted with the accuracy up to some background level) upon the energy is quasi-Lorentzian (see, e.g., the inset of Fig. 2c). The shape of the permeability coefficient in the vicinity of an arbitrary resonance main QSSs and the satellite QSSs is determined by a non-Lorentzian function. It depends essentially on the field energy $\Omega$ and the electron energy $E$ at a fixed electric intensity ( $\mathcal{E}$ or $U_{a c}$ ).

Fig. 3 displays dependences of the permeability coefficient on the electron energy at three fixed electromagnetic field energies for the vicinity of the energies of the second main QSS resonating with the first low-frequency satellite of the third main QSS (Fig. 3a) and for the vicinity of the energies of the third main QSS resonating with the first high-frequency satellite of the second main QSS (Fig. 3b). They are calculated for the electric intensity corresponding to the energy shift $U_{a c}=5 \mathrm{meV}$.
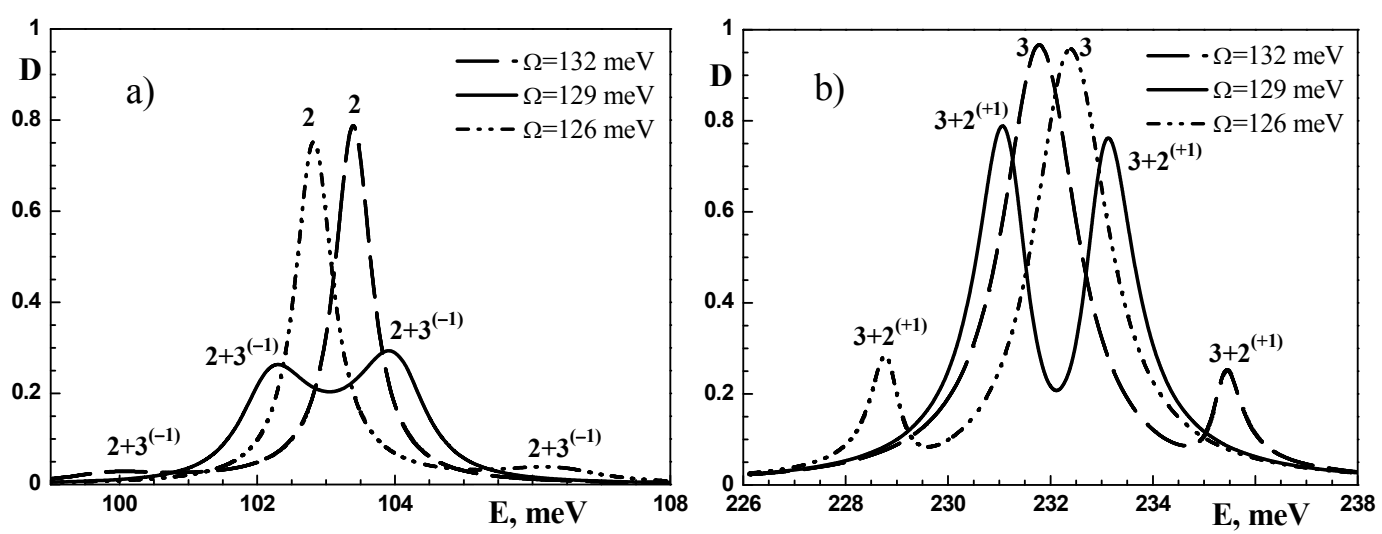

Fig. 3. Dependences of permeability coefficient $D$ on the electron energy $E$ for the cases when the electromagnetic field energy $\Omega$ is close to the difference between the energies of the third and the second QSSs in the vicinity of the second QSS (a) and the third QSS (b). Calculations are performed for $U_{a c}=5 \mathrm{meV}$.

One can see from Fig. 3 that, if the field energy is slightly smaller than the resonance one $\left(\Omega=132 \mathrm{meV}<E_{3}-E_{2}=129 \mathrm{meV}\right)$, the resonance energy of the first low-frequency satellite of the third QSS $\left(E_{3^{(-1)}}\right)$ is situated in the high-frequency region near the resonance energy of the second QSS $\left(E_{2}\right)$. At the same time, the resonance energy of the first high-frequency satellite of the second QSS $\left(E_{2^{(+1)}}\right)$ is situated in the low-frequency region near the resonance energy of the third QSS

$\left(E_{3}\right)$. If the field energy is slightly larger than the resonance one $\left(\Omega=132 \mathrm{meV}>E_{3}-E_{2}=129 \mathrm{meV}\right)$, the resonance energies of the satellite QSSs are situated in the low-frequency region of the second QSS and the high-frequency region of the third QSS, respectively. In the both cases the magnitudes of the satellite peaks of the permeability coefficient are smaller than the peaks of the corresponding main QSSs.

If the field energy is equal to the difference between the resonance energies of third and second QSS ( $\Omega=129 \mathrm{meV}$ ), then the permeability coefficient has a shape of two-humped curve with almost equal maxima in the vicinity of the both resonance energies $\left(E_{2}=103 \mathrm{meV}\right.$ and $E_{3}=232 \mathrm{meV}$ ). The both pairs of the QSSs are complex states corresponding to the electron in the second main state (with the energy $E_{2}$ ) bound with the first satellite state of the third QSS 
(with the energy $E_{3}-\Omega$ ), Fig. $3 \mathrm{~b}$, and in the third main state (with the energy $E_{3}$ ) bound with the first satellite state of the second QSS (with the energy $E_{2}+\Omega$ ), Fig. $3 \mathrm{a}$.

Thus, the arbitrary pairs of double peaks, which appear in the dependences of the permeability coefficient on the electron energy and are located in the vicinities of the main resonance energies of the nth and $n^{\prime}$ th QSSs represent manifestations of complex QSSs arising due to superposition of the main $n$th state with the first satellite one of the $n^{\prime}$ th QSS and the main $n^{\prime}$ th with the first satellite one of the nth QSS, respectively.

It is worthwhile to notice that the work [12] has not revealed the physical nature of the complex QSSs. Instead, it has only been told of the existence of two non-resonance peaks of the permeability coefficient for the two-barrier RTSs, because their maxima are shifted in the opposite directions with respect to the resonance energy.

\section{Conclusions}

Solving exactly the non-stationary Schrödinger equation, we have developed a quantummechanical theory of permeability coefficient for the two-barrier RTS for the case of electronic current interacting with the high-frequency electromagnetic field characterised with arbitrary magnitude of the electric intensity.

It is shown that, besides the electron QSSs with the main resonance energies, satellite QSSs appear in the spectrum, due to interaction with the electromagnetic field. Their spectrum contains all of possible harmonics (multiples by the field energy) located near every main resonance energy. If the energy of the high-frequency electromagnetic field is equal to the difference between any two electron resonance energies, two pairs of non-resonance transparency canals appear in the two-barrier RTS in the vicinity of both these resonance energies.

These peculiarities of quasi-stationary spectrum of the electrons interacting with the electromagnetic field in the two-barrier RTS change the active conductivity of this and the other complicated nanostructures. We remind that the latter structures represent basic elements of quantum cascade lasers and detectors, and so the peculiarities mentioned above would essentially influence the operation characteristics of these devices. While developing in future a theory of the active conductivity for the complicated nanostructures, we will take into consideration not only the main harmonics, as it has been done before, but the satellite harmonics, too.

\section{References}

1. Faist J, Capasso F, Sivco D L, Sirtori C, Hutchinson A L and Cho A Y, 1994. Quantum cascade laser. Science. 264: 553-556.

2. Faist J, Capasso F and Sirtori C, 1995. Vertical transition quantum cascade laser with Bragg confined excited state. Appl. Phys. Lett. 66: 538-540.

3. Berger V, French patent No 0109754 (2001).

4. Hofstetter D, Beck M and Faist J, 2002. Quantum-cascade-laser structures as photodetectors. Appl. Phys. Lett. 81: 2683-2685.

5. Elesin V F, 2005. High-frequency response of two-well nanostructure. JETP. 100: 116-125.

6. Elesin V F and Kateev I Yu, 2008. High-frequency properties of double-well nanostructures. Semiconductors. 42: 571-575.

7. Belyaeva I V, Golant E I and Pashkovskii A B, 1997. Resonant interaction of electrons with a high frequency electric field in two-barrier structures. Semiconductors. 31: 103-109.

Ukr. J. Phys. Opt. 2012, V13, №1 
8. Pashkovskii A B, 2005. Parity and abrupt broadening of resonance levels in triple-barrier structures. JETP Lett. 82: 210-214.

9. Tkach M V and Seti Yu O, 2010. Quasistationary electron states and the conductivity of a symmetric three-barrier resonance-tunnel structure. Ukr. J. Phys. 55: 798-807.

10. Golant E I and Pashkovskii A B, 1997. High quantum efficiency of intersubband transitions in coherent tunneling of electrons through asymmetric double-barrier structures. JETP. 85: $130-134$.

11. Golant E I and Pashkovskii A B, 1999. Application restrictions for two-level models of the resonance interaction of electrons with an alternating electric field in two-barrier structures. Theor. Math. Phys. 120: 1094-1101.

12. Pashkovskii A B, 2011. Splitting of the resonance levels of double-barrier structures in a high microwave electric field. JETP Lett. 93: 559-563.

Tkach M. V., Seti Ju. O. and Voitsekhivska O. M., 2012. Evolution of electron spectrum in symmetric two-barrier open nanostructure under the influence of strong electromagnetic field. Ukr.J.Phys.Opt. 13: 36 - 44.

Анотація. 3 використанням точного розв'язку повного рівняння Шредінгера розвинута теорія квазі-стаціонарних спектрів електронів, взаємодіючих з електромагнітним полем довільної інтенсивності у відкритих, двох-бар'єрних, резонансних, тунельних структурах. Показано, що крім квазі-стаціонарних електронних станів, що володіють звичайною резонансною енергією і шириною у спектрах виникають додаткові сателітні квазі-стаиіонарні стани з резонансними енергіями, розташованими біля енергій кожного з квазістаціонарних станів на енергетичних відстанях, що визначаються помноженням частот електромагнітного поля. 